\title{
Fusion Dynamics of Low-Energy Heavy-Ion Collisions for Production of Superheavy Nuclei
}

\author{
Xiao Jun Bao* \\ Department of Physics, Collaborative Innovation Center for Quantum Effects, and Key Laboratory of Low Dimensional \\ Quantum Structures and Quantum Control of Ministry of Education, Hunan Normal University, Changsha, China
}

One of the major motivations for low-energy heavy-ion collision is the synthesis of superheavy nuclei. Based on the following two main aspects, various theoretical and experimental studies have been performed to explore the fusion dynamical process of superheavy nuclei production. The first reason is to elucidate and analyze the synthesis mechanism of superheavy nuclei; the other is to search the favorable incident energy and the best combination of projectile and target to produce new superheavy elements and isotopes of superheavy elements.

Keywords: low-energy heavy-ion collisions, superheavy nuclei production, fusion dynamics, transport theory, TDHF model

OPEN ACCESS

Edited by:

Cedric Simenel,

Australian National University, Australia

Reviewed by:

Sait Umar

Vanderbilt University, United States

Nikolai Antonenko,

Joint Institute for Nuclear Research

(JINR), Russia

*Correspondence:

Xiao Jun Bao

baoxiaojun@hunnu.edu.cn

Specialty section:

This article was submitted to

Nuclear Physics,

a section of the journal

Frontiers in Physics

Received: 21 November 2019

Accepted: 16 January 2020

Published: 28 February 2020

Citation:

Bao XJ (2020) Fusion Dynamics of Low-Energy Heavy-lon Collisions for

Production of Superheavy Nuclei.

Front. Phys. 8:14

doi: 10.3389/fphy.2020.00014

\section{INTRODUCTION}

The maximum mass and charge of a nucleus is a long-standing fundamental problem in nuclear physics $[1,2]$. Pioneer studies have theoretically predicted the "island of stability" of superheavy nuclei (SHN). The macroscopic-microscopic models predicted ${ }^{298} \mathrm{Fl}$ to be the double magic nucleus [3-7]. However, results of the self-consistent models showed that the closed shell of $Z=114$ becomes weaker, and $Z=114$ is replaced by $Z=120$ or 126 [8-13].

The production process of superheavy nuclei is a very complicated dynamical problem [14]. Many theoretical models have been developed to explain the experimental data. On one hand, the synthesis mechanism of superheavy nuclei needs to be elucidated [15-21]. Different approaches are devoted to calculate and analyze the fusion probability and the distribution of quasifission fragments [15-41]. However, none of them has absolute advantage. On the other hand, in order to produce the new superheavy elements, or isotopes of superheavy elements, the favorable incident energy and the best combination of projectile and target should be evaluated.

The extended nuclear landscape allows us to investigate the nuclear structure of superheavy nuclei and the nuclear reaction mechanism. To search for the optimal condition of synthesis, the influence of the entrance channel $[29,42,43]$ and the isospin of heavy colliding nuclei [44-46] on the evaporation residual cross section have been studied systematically in many works. The predictions of the possible way to synthesize the new superheavy elements $Z=119$ and 120 have also been carried out [17, 47-52].

\section{EXPERIMENTAL PROGRESS}

Producing superheavy nuclei in the laboratory is one of the major motivations of low-energy heavy-ion physics [1, 2, 53-55]. Over the past 30 years, great progress has been achieved for superheavy nuclei production in experimental studies [53-55]. The experimental trends $\alpha$ decay half-lives, and the evaporation residue cross sections of the superheavy nuclei show 
that the stability of superheavy nuclei increases as the neutron number approaches the closed neutron shell closure $N=184$. However, the location of the "island of stability" has not been determined by experiment. Up until now, based on the fusionevaporation reaction, the superheavy nuclei with charge numbers in the range of $Z=104118$ have been synthesized successfully.

The superheavy elements $Z=107-112$ was first synthesized by using the cold fusion reactions $[1,53,54,56,57]$. The excitation energy range of the formed compound nucleus was $10-18 \mathrm{MeV}$. The measurement of the evaporation residue cross section decreased dramatically from $Z=107$ to $Z=113$. Moreover, the final evaporated residual nuclei were extremely neutron deficient. Experiments of producing superheavy nuclei by cold fusion have been repeated and verified by other laboratories [54, 58, 59].

The ${ }^{48} \mathrm{Ca}$-induced hot-fusion reactions were used to synthesize $Z=112-118$ superheavy nuclei in experiment $[54,55,60]$. From the measurement of evaporation residue cross sections, we found that there was no significant difference from $Z=112-118$, and the values of the evaporation residual cross sections were all in the order of picobarn. Experiments based on hot fusion for synthesizing $Z=112$ and 114-117 superheavy nuclei have been verified by other laboratories [60].

To search for the optimal condition of the superheavy nuclei production, various experiments have been performed to study the entrance channel effect on the evaporation residual cross section [61-65]. Recently, the isospin effect of the target nucleus on the evaporation residue cross section has been explored [6668]. Some laboratories have also attempted to synthesize the $Z=$ 119 and 120 superheavy elements by using hot fusion $[69,70]$.

Experimentally, the measurement of fusion probability is required to distinguish quasifission between fusion-fission and fast fission [71-76]. The experimental characteristics of the quasifission process are different from the fusion-fission process [77]. Therefore, it is important to distinguish the fusion and quasifission fragments for a better understanding of the fusion mechanism.

\section{THEORETICAL DESCRIPTION OF FUSION REACTIONS}

Theoretically, the synthesis process of superheavy nuclei can be divided into three stages [39]. A schematic diagram for this process is shown in Figure 1. The first stage is the capture process, which can be evaluated by the capture cross section. The second stage is that the dinuclear system evolves from the touching configuration to the formation of the compound nucleus, which can be evaluated by the fusion probability. The last stage is where the excited compound nucleus cools down through emitting neutrons or fission, and this can be evaluated by the survival probability. Finally, a very small evaporation residue cross section is obtained for the superheavy nuclei production. The evaporation residue cross section can be expressed as [39],

$$
\sigma_{E R}\left(E_{c . m .}\right)=\sum_{J} \sigma_{c a p}\left(E_{c . m .}, J\right) P_{C N}\left(E_{c . m .}, J\right) W_{\text {sur }}\left(E_{c . m .}, J\right)
$$

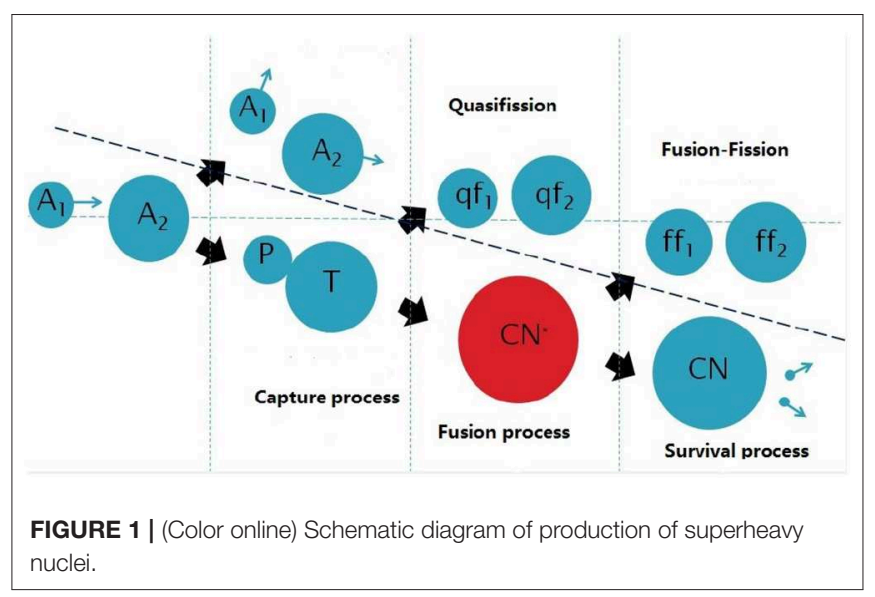

where $E_{c . m}$ is the incident energy in the center-of-mass frame.

\subsection{Capture Cross Sections}

For the low-energy heavy-ion collision, the capture cross section from the sub-barrier region to above the Coulomb barrier is an important issue for theoretical and experimental studies [7883]. One of reasons is that the overall uncertainties in predicting superheavy nuclei production are associated with the calculations of capture cross sections [50, 84, 85].

The capture process is closely related to the nuclear structure of the interacting nuclei [86-97]. This is because the nucleusnucleus potential contains nuclear structure information. To precisely describe the measurements of capture cross sections, the nucleus-nucleus interaction potential is the most important input quantity. In addition, the heavy-ion capture process is intimately linked to nuclear deformation [87, 88]. Thus, the nuclear deformation must be reliable to some extent.

Theoretically, the capture cross section is one of the important components in the synthesis of superheavy nuclei. The capture cross section have been explored extensively [82-84] from light to superheavy by averaging the penetration probability over barrier heights. Most of them have tested a number of experimental data of capture cross sections, however, these experimental data do not contain the capture cross sections of superheavy nuclei [84]. Therefore, it is very important to examine carefully the capture process for the study of the synthesis mechanism of superheavy nuclei.

Usually, the capture cross section $\sigma_{c a p}$ is mainly calculated with an empirical coupled-channel approach for the superheavy nuclei production $[17,44-52]$. From a theoretical point of view, one of the powerful methods is to solve coupled-channels equations numerically. This may help us to understand the influence of the couplings between nuclear intrinsic degrees of freedom and the relative motion on capture cross sections.

Recently, the quantum diffusion approach [98-101] has also been used to calculate capture cross sections. This model takes into consideration the influence of fluctuations and dissipation effects on capture cross sections. The nuclear deformation effects and mutual orientations of the colliding nuclei are taken into account through using a double folding potential, and 
the influence of two neutrons transfer onto the sub-barrier capture through the change of the deformations of the colliding nuclei [100].

Another powerful theoretical tool is to calculate the capture cross section by the time-dependent Hartree Fock (TDHF) method. Recently, the pioneering work of studying capture cross section based on TDHF has been completed for the ${ }^{40} \mathrm{Ca}+{ }^{238} \mathrm{U}$ reaction [102]. In addition, Umar et al. found that the capture cross sections calculated by TDHF method agreed with the experimental data within $20 \%$ [103].

\subsection{Fusion Dynamics}

In order to explain the fusion dynamics process (the second stage), various theoretical approaches and models have been developed. The simplification can be made in different ways, and, as a result, we can obtain different theoretical pictures for the same real nuclear process. Some of the models can be roughly divided into two types. The first type is based on transport equations to describe the fusion dynamics process [15-41]. The second one is based on the time-dependent Hartree Fock method to describe the mainly experimental features in the process of fusion dynamics [104-115].

The first approach is that the multitude of degrees of freedom are decomposed into a dominating collective degree of freedom and other degrees (non-collective) of freedom. Therefore, the dissipative processes are introduced to account for the coupling between the collective motions and the intrinsic motions of the freedom of the system. Many models based on transport equations have been developed, and they assumed that the main characteristics of fusion dynamics process can be described by using the main collective degrees of freedom.

On the one hand, after eliminating the intrinsic motion, a stochastic equation can be derived theoretically. Many models adopted the Langevin forces (Langevin equation) to describe stochastic characteristics of the coupling between collective motions and intrinsic degrees of freedom. One can calculate a bundle of trajectories by solving a stochastic equation $[16,17,28$, $31,35,36]$.

On the other hand, through eliminating the intrinsic degrees of freedom, a diffusion equation can be derived theoretically to describe the distribution of collective degrees of freedom in the phase space $[19-21,27,29,29,30,32,41-43,46,47,49,51,116$, 117]. Diffusion equations (the master equation, Smoluchowski equation, etc.,) may be used to describe the transport process of collective degrees of freedom in phase space.

The second approach is the time-dependent Hartree Fock method. The basic idea of this method is that the mean field produced by all nucleons not only determines the intrinsic motion of a single particle but also describes the evolution characteristics of collective degrees. TDHF calculations may be used to compute the ratio of fusion cross sections to capture cross sections. In addition, the TDHF method may be used to explore the effect of the orientation of the projectile and the target at the contact point, and the role of the nuclear shell structure and tensor force [104-115].

\subsection{Fusion Mechanism}

For the real fusion dynamics process, a theoretical model may be considered as a collection of theoretical assumptions. Up to now, there have been proposed fusion mechanisms that are incompatible with the compound nucleus formation. One assumption is that all the nucleons are immediately collectivized into one superdeformed mononucleus. Then, the dynamic evolution behavior of the superdeformed mononucleus can be described by the equation of motion or transport theory [15-17, $33,34,118]$. The macroscopic dynamical model is the first model to describe the fusion mechanism based on the idea of forming one superdeformed mononucleus [15]. However, it encountered serious difficulties in attempts to describe evaporation residue cross sections for the synthesis of superheavy nuclei.

As the macroscopic dynamical models, the same approximations are used in the fluctuation-dissipation model $[16,118]$, the two-step model [17], and the fusion-by-diffusion model $[33,34]$. But two significant improvements are taken into account for the description of the fusion-dynamics mechanism: shell effects in the calculation of the potential energy surface of the reaction system and statistical fluctuations in the interaction of colliding nuclei. These improvements permit one to describe the evaporation residue cross section of superheavy nuclei, the mass distribution of quasifission, and fusion-fission products [16, 17, 33, 34, 118, 119].

Another assumption is that two touching nuclei always keeps its own identity with their ground state characteristics and deformations (dinuclear system model) [19-21, 29], fusion is achieved by means of nucleon transfer. However, the real situation is due to strong Coulomb and nuclear interactions between projectile and target; the dinuclear system should be gradually deformed $[30,48]$. This assumption has recently been improved upon. The coupling of the deformations evolution of project and target and the nucleon transfer has been studied numerically $[120,121]$. The calculated results for the cold and hot fusion reactions by using the dinuclear system model match well with the available experimental data $[20,27,29,29,30$, $32,41-43,46,47,49,51,116,117]$. The fusion probability and the distribution of the quasifission fragments can be reasonably described based on the dinuclear system model [120-122].

A new fusion mechanism on compound nucleus formation was proposed by Zagrebaev [18]. The concept of a nucleon collectivization model assumes that two nuclei gradually lose their individualities through increasing the number of collectivized nucleons [18]; the reliability of the theoretical hypothesis needs further demonstration [123]. The nucleon collectivization model allows us to describe reasonably the fusion probability as well as the charge and mass distributions of the quasifission products [124].

\subsection{Selection of Collective Degree and Calculation of Related Input Quantity in Transport or Diffusion Equations}

To theoretically describe fusion dynamics and the mechanism based on transport equations as mentioned above, one needs to assume that several important degrees of freedoms can be 
used to describe the main characteristics of fusion dynamics process [15-21]. These important degrees of freedoms include the distance between the nuclear centers, the neutron and proton asymmetries of projectile-target combinations, deformations, and corresponding orientation effects, which influence the dynamics from touching the configuration to the compound nucleus.

Because equations of motion contain time derivatives up to the second order, there are three quantities in each equations of motion. The first quantity is the conservative potential. The second and third are the friction tensor (friction force) and the inertia tensor (inertia parameter), respectively. For the conservative potential, two different approaches have been taken into account to calculate the potential energy surface. The two assumptions of calculating potential energy surface are frozen density or sudden approximation [19-21] and the adiabatic approximation [15-18].

Recently, Diaz-Torres showed that the gradual transition of potential energy surface from the diabatic to the adiabatic should be more realistic for describing the fusion or quasifission $[125,126]$. In addition, one needs to consider how the shell structure evolutes with excitation energy and deformation. The excitation energy dissipated from kinetic energy of relative motion makes the individual shell structure of nuclei become damped [127-131], and deformation tends to be spherical [132]. Thus, the dynamical potential energy surface has to be further studied. However, a small amount of research work has involved the shell correction energy employed in the fusion process being temperature dependent [133] and the potential energy surface from diabatic approximation to adiabatic approximation to describe the whole dynamic evolution process.

The dissipation tensor arises from the distinction between collective motion and intrinsic motion. The dissipation tensor accounts for the coupling between the collective degrees of freedom and other degrees (non-collective) of freedom. When equations of motion or stochastic equations are used to describe the dynamic process, the friction coefficients are mainly treated by the phenomenological approaches for the description of the fusion dynamics process $[16,124]$.

The inertial tensor describes the response of the system to small changes in the collective degrees of freedom. The macroscopic approach, macroscopic-microscopic approach, and microscopic approach are used to calculate the inertia tensor of the fission dynamics process [134-137]. However, in the low-energy heavy-ion collisions process, it seems that the proper calculation on inertial parameters has not been paid enough attention compared to the fission dynamics process. In the stochastic equation, the inertia parameter is calculated by the Werner-Wheeler approach $[124,127]$. In the diffusion equation, the inertia parameter is treated as a reduced mass of relative motion [138]. From the theoretical point of view, the inertia parameters and friction coefficient of theoretical calculations have to match our understanding of the potential energy surface in the transport equations.

\subsection{Survival Probabilities}

The last important factor is the survival probability of the compound nucleus against fission in the deexcitation process. For exciting compound nuclei, there are two methods to describe the fission process: the statistical approach and the dynamical approach. Based on the statistical model, two different approaches are taken into account for the excitation energy dependent shell structure. The first one is to introduce the influence of excitation energy on the shell structure through the energy level density parameter [139]. The second one is to ensure the excitation energy-dependent shell effect is taken into account by the effective potential energy surface [140].

The uncertainty of survival probability calculation based on statistical model mainly comes from two aspects. On one hand, a number of approximations are adapted in the calculation of survival probability. One the other hand, the survival probabilities for the $x n$ evaporation channels are very sensitive to the model input. The level densities, fission barriers, neutronseparation energies, as well as the transmission coefficients have to be known with sufficient accuracy. Only systematic calculations based on the same assumptions and parameters can help to confirm the validity and reliability of the theoretical approximations and input quantities $[28,117]$.

\section{THE OPTIMUM PROJECTILE-TARGET COMBINATION AND BOMBARDING ENERGY}

\subsection{Influence of Entrance Channel on ERCSs}

Systematic studies of the existing experimental ERCSs are helpful to reveal reaction mechanisms. In addition, searching the optimal combination of the projectile and target and the range of the favorable beam energy is essential.

Some work systematically studies the influence of the neutron number of a target or projectile on the evaporation residue cross section [32, 36, 44-46]. Many researchers have found that the fusion probability and survival probability are sensitive to the neutron number of the target or projectile nuclei $[32,36,44-$ 46]. For ${ }^{48} \mathrm{Ca}$-induced hot fusion, the excess neutron of target nucleus is beneficial to the increase of the evaporation residue cross section of the synthesized superheavy nuclei $[32,45,46]$.

Using a different combination of projectile and target to produce the same compound nucleus may help us to reveal the effect of the ground-state deformations, the reaction $Q$ value, the asymmetry of charge and mass of target and projectile, and the Coulomb barrier on the evaporation residual cross section $[33,138,141]$. The results calculated by Liu et al. show that the $Q$ value of reaction has a significant effect on the capture cross section and fusion probability [142].

\subsection{The ERCS of Production Using Radioactive Beams}

In order to investigate the possibility of neutron-rich superheavy nuclei production with radioactive beams, some calculations have been made $[25,116,143,144]$. The evaporation residue 
cross section by using radioactive beams are comparable with a stable beam for some superheavy nuclei production [116, 143]. However, the choice of reaction channels is determined by the product of beam intensity and evaporation residue cross section [143].

The intensities of stable beams, in most of the cases, are significantly larger than those of the radioactive beams [143], and the results have shown that the calculated evaporation residue cross section based on stable beam is more favorable for the production of many superheavy nuclei [116, 143]. In addition, due to small evaporation residue cross sections and low radioactive beam intensities, the synthesis of higher charge number superheavy nuclei by using the neutronrich radioactive beams seems impossible based on today's experimental conditions [116, 143].

\subsection{Prediction ERCS $Z=119$ and 120}

By comparing evaporation residue cross sections for production $Z=119$ and 120 , we found that the calculations from different models were obviously different $[17,47-52,124]$. On one hand, the survival probability of the compound nucleus was very sensitive to the fission barrier. However, the difference in fission barriers of superheavy nuclei calculated by different models was obvious not only due to the absolute values but also the trends with charge number $Z[2,85]$. On the other hand, due to different assumptions, the fusion probability calculated by different models was significantly different for synthesis $Z=119$ and 120 [84].

According to our calculation and other theoretical predictions, almost all the models that predicted evaporation residue cross sections to produce superheavy new element $Z=119$ are generally greater than those in producing $Z=120[17,47-52]$.

\section{THE FUTURE}

The synthesis of SHN in the laboratory has made great progress, and all the elements up to $Z=118$ have been synthesized successfully. However, the location of the island of stability has not been confirmed in experiments. Although many theoretical approaches were used to study the fusion mechanism, there are still many problems that have not been solved properly.

\section{REFERENCES}

1. Hofmann S, Münzenberg G. The discovery of the heaviest elements. Rev Mod Phys. (2000) 72:733. doi: 10.1103/RevModPhys.72.733

2. Giuliani SA, Matheson Z, Nazarewicz W, Olsen E, Reinhard PG, Sadhukhan J, et al. Colloquium: superheavy elements: oganesson and beyond. Rev Mod Phys. (2019) 91:011001. doi: 10.1103/RevModPhys.91.011001

3. Sobiczewski A, Gareev FA, Kalinkin BN. Closed shells for Z>82 and $\mathrm{N}>126$ in a diffuse potential well. Phys Lett. (1966) 22:500. doi: 10.1016/0031-9163(66)91243-1

4. Mosel U, Greiner W. On the stability of superheavy nuclei against fission. $Z$ Phys. (1969) 222:261. doi: 10.1007/BF01392125

5. Nilsson SG, Tsang CF, Sobiczewski A, Szymanski Z, Wycech S, Gustafson $\mathrm{C}$, et al. On the nuclear structure and stability of heavy and superheavy elements. Nucl Phys A. (1969) 131:1. doi: 10.1016/0375-9474(69)90809-4
In my opinion, the transport theory is the least developed one due to three factors: (i) the neck formation itself and the relationship between dynamic deformation and neck formation should be included to improve the theoretical description of fusion dynamic mechanism; (ii) the gradual transition of the potential energy surface from the diabatic approximation to the adiabatic approximation needs to be further explored; and (iii) the further study of inertia and damping coefficients to match our understanding of the potential energy surface in the transport equations should be performed.

\section{SUMMARY}

The evaporation residue cross section of superheavy nuclei depends on three factors: the capture cross section $\sigma_{\text {cap }}$, the fusion probability $P_{C N}$, and the survival probability $W_{\text {sur }}$. We found that the reasonable description of the capture cross section near the Coulomb barrier is very important. The coupled channel or TDHF can be better approached to calculate the value, especially for superheavy nuclei calculations.

I think the fusion probability $P_{C N}$ for producing superheavy nuclei is still not well understood. Not only the magnitude of the fusion probability $P_{C N}$ but also the dependence of $P_{C N}$ on the excitation energy and the entrance channel are lacking in clarity. The fusion mechanism must be further studied.

The uncertainty of survival probability calculation based on a statistical model mainly comes from theoretical approximations and input quantities. Systematic calculations based on the same assumptions and parameters can help to confirm the validity and reliability of the theoretical approximations and input quantities.

\section{AUTHOR CONTRIBUTIONS}

The author confirms being the sole contributor of this work and has approved it for publication.

\section{FUNDING}

This work was supported by the National Natural Science Foundation of China (Grants 11705055) and Hunan Provincial Natural Science Foundation of China (2018JJ3324).

6. Moller P, Nix JR, Myers WD, Swiatecki WJ. Nuclear ground-state masses and deformations. At Data Nucl Data Tables. (1995) 59:185. doi: 10.1006/adnd.1995.1002

7. Smolańczuk R. Properties of the hypothetical spherical superheavy nuclei. Phys Rev C. (1997) 56:812. doi: 10.1103/PhysRevC.56.812

8. Decharge J, Berger JF, Dietrich K, Weiss MS. Superheavy and hyperheavy nuclei in the form of bubbles or semi-bubbles. Phys Lett B. (1999) 451:27582. doi: 10.1016/s0370-2693(99)00225-7

9. Bender M, Nazarewicz W, Reinhard PG. Shell stabilization of super- and hyperheavy nuclei without magic gaps. Phys Lett B. (2001) 515:42-8. doi: 10.1016/S0370-2693(01)00863-2

10. Ring P. Relativistic mean field theory in finite nuclei. Prog Part Nucl Phys. (1996) 37:193-263. doi: 10.1016/0146-6410(96)00054-3

11. Meng J, Toki H, Zhou SG, Zhang SQ, Long WH, Geng LS. Relativistic continuum Hartree Bogoliubov theory for ground-state 
properties of exotic nuclei. Prog Part Nucl Phys. (2006) 57:470-563. doi: 10.1016/j.ppnp.2005.06.001

12. Li JJ, Long WH, Margueron J, Van Giai N. Superheavy magic structures in the relativistic HartreeCFockCBogoliubov approach. Phys Lett B. (2014) 732:169-73. doi: 10.1016/j.physletb.2014.03.031

13. Cwiok S, Dobaczewski J, Heenen PH, Magierski P, Nazarewicz W. Shell structure of the superheavy elements. Nucl Phys A. (1996) 611:211-46. doi: 10.1016/S0375-9474(96)00337-5

14. Swiatecki WJ. Three lectures on macroscopic aspects of nuclear dynamics. Prog Part Nucl Phys. (1980) 4:383. doi: 10.1016/0146-6410(80)90014-9

15. Swiatecki WJ. The dynamics of the fusion of two nuclei. Nucl Phys A. (1982) 376:275. doi: 10.1016/0375-9474(82)90065-3

16. Aritomo Y, Wada T, Ohta M, Abe Y. Fluctuation-dissipation model for synthesis of superheavy elements. Phys Rev C. (1999) 59:796. doi: 10.1103/PhysRevC.59.796

17. Shen CW, Abe Y, Boilley D, Kosenko G, Zhao EG. Isospin dependence of reactions $48 \mathrm{Ca}+243-251 \mathrm{Bk}$. Int. J. Mod. Phys. E. (2008) 17:66. doi: 10.1142/S0218301308011768

18. Zagrebaev VI. Synthesis of superheavy nuclei: nucleon collectivization as a mechanism for compound nucleus formation. Phys Rev C. (2001) 64:034606. doi: 10.1103/PhysRevC.64.034606

19. Adamian GG, Antonenko NV, Scheid W, Volkov VV. Treatment of competition between complete fusion and quasifission in collisions of heavy nuclei. Nucl Phys A. (1997) 627:361. doi: 10.1016/S0375-9474(97)00605-2

20. Feng ZQ, Jin GM, Fu F, Li JQ. Production cross sections of superheavy nuclei based on dinuclear system model. Nucl Phys A. (2006) 771:50. doi: 10.1016/j.nuclphysa.2006.03.002

21. Diaz-Torres A, Adamian GG, Antonenko NV, Scheid W. Quasifission process in a transport model for a dinuclear system. Phys Rev C. (2001) 64:024604. doi: 10.1103/PhysRevC.64.024604

22. Zagrebaev V, Greiner W. Synthesis of superheavy nuclei: a search for new production reactions. Phys Rev C. (2008) 78:034610. doi: 10.1103/PhysRevC.78.034610

23. Smolańczuk R. Synthesis of transactinide nuclei in cold fusion reactions using radioactive beams. Phys Rev C. (2010) 81:067602. doi: 10.1103/PhysRevC.81.067602

24. Wang N, Tian J, Scheid W. Systematics of fusion probability in hot fusion reactions. Phys Rev C. (2011) 84:061601(R). doi: 10.1103/PhysRevC.84.061601

25. Wu ZH, Zhu L, Li F, Yu XB, Su J, Guo CC. Synthesis of neutron-rich superheavy nuclei with radioactive beams within the dinuclear system model. Phys Rev C. (2018) 97:064609. doi: 10.1103/PhysRevC.97.064609

26. Shen CW, Kosenko G, Abe Y. Two-step model of fusion for the synthesis of superheavy elements. Phys Rev C. (2002) 66:061602. doi: 10.1103/PhysRevC.66.061602

27. Wang N, Li JQ, Zhao EG. Orientation effects of deformed nuclei on the production of superheavy elements. Phys Rev C. (2008) 78:054607. doi: 10.1103/PhysRevC.78.054607

28. Siwek-Wilczyńska K, Cap T, Kowal M, Sobiczewski A, Wilczyński J. Predictions of the fusion-by-diffusion model for the synthesis cross sections of $\mathrm{Z}=114-120$ elements based on macroscopic-microscopic fission barriers. Phys Rev C. (2012) 86:014611. doi: 10.1103/PhysRevC.86.014611

29. Nasirov AK, Giardina G, Mandaglio G, Manganaro M, Hanappe F, Heinz $S$, et al. Quasifission and fusion-fission in reactions with massive nuclei: Comparison of reactions leading to the $Z=120$ element. Phys. Rev. C. (2009) 79:024606. doi: 10.1103/PhysRevC.79.024606

30. Bao XJ, Gao Y, Li JQ, Zhang HF. Influence of the nuclear dynamical deformation on production cross sections of superheavy nuclei. Phys Rev C. (2015) 91:011603(R). doi: 10.1103/PhysRevC.91.011603

31. Litnevsky VL, Pashkevich VV, Kosenko GI, Ivanyuk FA. Description of synthesis of super-heavy elements within the multidimensional stochastic model. Phys Rev C. (2014) 89:034626. doi: 10.1103/PhysRevC.89.034626

32. Adamian GG, Antonenko NV, Scheid W. Isotopic trends in the production of superheavy nuclei in cold fusion reactions. Phys Rev C. (2004) 69:011601. doi: 10.1103/PhysRevC.69.011601

33. Liu $\mathrm{ZH}, \mathrm{Bao}$ JD. Isotopic dependence of the evaporation residue cross section in the synthesis of superheavy nuclei. Phys Rev C. (2007) 76:034604. doi: 10.1103/PhysRevC.76.034604
34. Liu ZH, Bao JD. Synthesis of superheavy element 120 via50Ti+ACf hot fusion reactions. Phys Rev C. (2009) 80:054608. doi: 10.1103/PhysRevC.80.05 4608

35. Zagrebaev VI, Karpov AV, Greiner W. Possibilities for synthesis of new isotopes of superheavy elements in fusion reactions. Phys Rev C. (2012) 85:014608. doi: 10.1103/PhysRevC.85.014608

36. Cap T, Wilczynska KS, Kowal M, Wilczynski J. Calculations of the cross sections for the synthesis of new 293-296118 isotopes in 249-252Cf(48Ca,xn) reactions. Phys Rev C. (2013) 88:037603. doi: 10.1103/PhysRevC.88.037603

37. Wang N, Zhao EG, Scheid W. Synthesis of superheavy nuclei with $\mathrm{Z}=118$ in hot fusion reactions. Phys Rev C. (2014) 89:037601. doi: 10.1103/PhysRevC.89.037601

38. Li W, Wang N, Jia F, Xu H, Zuo W, Li JQ et al. Fusion probability in heavy-ion collisions by a dinuclear-system model. J Phys G Nucl Phys. (2006) 32:1143. doi: 10.1209/epl/i2003-00622-0

39. Antonenko N, Cherepanov E, Nasirov A, Permjakov V, Volkov V. Competition between complete fusion and quasi-fission in reactions between massive nuclei. The fusion barrier. Phys Lett B. (1993) 319:425. doi: 10.1016/0370-2693(93)91746-A

40. Antonenko NV, Cherepanov EA, Nasirov AK, Permjakov VP, Volkov VV. Compound nucleus formation in reactions between massive nuclei: fusion barrier. Phys Rev C. (1995) 51:2635. doi: 10.1103/PhysRevC.51.2635

41. Huang MH, Gan ZG, Feng ZQ, Zhou XH, Li JQ. Neutron and proton diffusion in fusion reactions for the synthesis of superheavy nuclei. Chin Phys Lett. (2008) 25:1243. doi: 10.1088/0256-307X/25/4/022

42. Nasirov A, Fukushima A, Toyoshima Y, Aritomo Y, Muminov A, Kalandarov $\mathrm{S}$, et al. The role of orientation of nucleus symmetry axis in fusion dynamics. Nucl Phys A. (2005) 759:342-69. doi: 10.1016/j.nuclphysa.2005. 05.152

43. Hong J, Adamian GG, Antonenko NV. Possibilities of production of transfermium nuclei in charged-particle evaporation channels. Phys Rev C. (2016) 94:044606. doi: 10.1103/PhysRevC.94.044606

44. Feng ZQ, Jin GM, Li JQ, Scheid W. Formation of superheavy nuclei in cold fusion reactions. Phys Rev C. (2007) 76:044606. doi: 10.1103/PhysRevC.76. 044606

45. Mandaglio G, Giardina G, Nasirov AK, Sobiczewski A. Investigation of the 48Ca+249-252Cf reactions synthesizing isotopes of the superheavy element 118. Phys Rev C. (2012) 86:064607. doi: 10.1103/PhysRevC.86.064607

46. Bao XJ, Gao Y, Li JQ, Zhang HF. Isotopic dependence of superheavy nuclear production in hot fusion reactions. Phys Rev C. (2015) 92:034612. doi: 10.1103/PhysRevC.92.034612

47. Liu ZH, Bao JD. Possibility to produce element 120 in the $54 \mathrm{Cr}+248 \mathrm{Cm}$ hot fusion reaction. Phys Rev C. (2013) 87:034616. doi: 10.1103/PhysRevC.87. 034616

48. Wang N, Zhao EG, Scheid W, Zhou SG. Theoretical study of the synthesis of superheavy nuclei with $\mathrm{Z}=119$ and 120 in heavy-ion reactions with trans-uranium targets. Phys Rev C. (2012) 85:041601(R). doi: 10.1103/PhysRevC.85.041601

49. Zhu L, Xie WJ, Zhang FS. Production cross sections of superheavy elements $\mathrm{Z}=119$ and 120 in hot fusion reactions. Phys Rev C. (2014) 89:024615. doi: 10.1103/PhysRevC.89.024615

50. Bao XJ, Guo SQ, Li JQ, Zhang HF. Influence of hexadecapole deformation on production cross sections of superheavy nuclei. J Phys G Nucl Part Phys. (2016) 43:125105. doi: 10.1088/0954-3899/43/12/125105

51. Huang MH, Zhang ZY, Gan ZG, Zhou XH, Li JQ, Scheid W. Dynamical deformation in heavy ion collisions and formation of superheavy nuclei. Phys Rev C. (2011) 84:064619. doi: 10.1103/PhysRevC.84.0 64619

52. Wang $\mathrm{N}$, Liu $\mathrm{M}$, Wu XZ. Modification of nuclear mass formula by considering isospin effects. Phys Rev C. (2010) 81:044322. doi: 10.1103/PhysRevC.81. 044322

53. Hofmann S. Synthesis of superheavy elements by cold fusion. Radiochim Acta. (2011) 99:405. doi: 10.1524/ract.2011.1854

54. Kosuke M, Kouji M, Daiya K, Takahiro A, Sin-ichi G, Hiromitsu H, et al. Experiment on Synthesis of an Isotope 277112 by $208 \mathrm{~Pb}+70 \mathrm{Zn}$ Reaction. J. Phys. Soc. Japan. (2007) 76:043201. doi: 10.1143/JPSJ.76. 043201 
55. Oganessian YT. Synthesis of the heaviest elements in 48Ca-induced reactions. Radiochim Acta. (2011) 99:429. doi: 10.1524/ract.2011.1860

56. Münzenberg G. Recent advances in the discovery of transuranium elements. Rep Prog Phys. (1988) 51:57. doi: 10.1088/0034-4885/51/1/002

57. Hofmann S. New elements-approaching $\mathrm{Z}=114$. Rep Prog Phys. (1998) 61:639.

58. Kosuke M, Kouji M, Daiya K, Takahiro A, Sin-ichi G, Hiromitsu $\mathrm{H}$, et al. Experiment on the synthesis of element 113 in the reaction 209Bi(70Zn,n)278113. J Phys Soc Jpn. (2004) 73:2593. doi: 10.1143/JPSJ.73.2593

59. Morita K, Morimoto K, Kaji D, Haba H, Ozeki K, Kudou Y, et al. New result in the production and decay of an isotope, 278113, of the 113th Element. $J$ Phys Soc Jpn. (2012) 81:103201. doi: 10.1143/JPSJ.81.103201

60. Oganessian YT, Utyonkov VK. Super-heavy element research. Rep Prog Phys. (2015) 78:036301. doi: 10.1088/0034-4885/78/3/036301

61. Dvorak J, Bruchle W, Dullmann Ch E, Dvorakova Z, Eberhardt K, Eichler R, et al. Cross section limits for the $248 \mathrm{Cm}(25 \mathrm{Mg}, 4 \mathrm{n}-5 \mathrm{n}) 268,269 \mathrm{Hs}$ reactions. Phys Rev C. (2009) 79:037602. doi: 10.1103/PhysRevC.79.0 37602

62. Graeger R, Ackermann D, Chelnokov M, Chepigin V, Dullmann Ch. E, Dvorak J, et al. Experimental study of the 238U(36S,3-5n)269-271Hs reaction leading to the observation of 270 Hs. Phys Rev C. (2010) 8:061601(R). doi: 10.1103/PhysRevC.81.061601

63. Nishio K, Hofmann S, Heberger FP, Ackermann D, Antalic S, Aritomo Y, et al. Nuclear orientation in the reaction $34 \mathrm{~S}+238 \mathrm{U}$ and synthesis of the new isotope $268 \mathrm{Hs}$. Phys Rev C. (2010) 82:024611. doi: 10.1103/PhysRevC.82.024611

64. Oganessian Yu Ts, Utyonkov VK, Abdullin FS, Dmitriev SN, Graeger R, Henderson RA, et al. Synthesis and study of decay properties of the doubly magic nucleus $270 \mathrm{Hs}$ in the $226 \mathrm{Ra}+48 \mathrm{Ca}$ reaction. Phys Rev C. (2013) 87:034605. doi: 10.1103/PhysRevC.87.034605

65. Dvorak J, Bruchle W, Chelnokov M, Dullmann Ch E, Dvorakova $\mathrm{Z}$, Eberhardt $\mathrm{K}$, et al. Observation of the $3 \mathrm{n}$ evaporation channel in the complete hot-fusion reaction $26 \mathrm{Mg}+248 \mathrm{Cm}$ leading to the new superheavy nuclide 271 Hs. Phys Rev Lett. (2008) 100:132503. doi: 10.1103/PhysRevLett.100.132503

66. Utyonkov VK, Brewer NT, Oganessian Yu Ts, Rykaczewski KP, Abdullin F, Dmitriev SN, et al. Experiments on the synthesis of superheavy nuclei 284Fl and $285 \mathrm{Fl}$ in the 239,240Pu+48Ca reactions. Phys Rev C. (2015) 92:034609. doi: 10.1103/PhysRevC.92.034609

67. Brewer NT, Utyonkov VK, Rykaczewski KP, Oganessian Yu Ts, Abdullin F, Boll RA, et al. Search for the heaviest atomic nuclei among the products from reactions of mixed-Cf with a 48Ca beam. Phys Rev C. (2018) 98:024317. doi: 10.1103/PhysRevC.98.024317

68. Nishio K, Mitsuoka S, Nishinaka I, Makii H, Wakabayashi Y, Ikezoe $\mathrm{H}$, et al. Fusion probabilities in the reactions $40,48 \mathrm{Ca}+238 \mathrm{U}$ at energies around the Coulomb barrier. Phys Rev C. (2012) 86:034608. doi: 10.1103/PhysRevC.86.034608

69. Oganessian YT, Utyonkov VK, Lobanov Yu V, Abdullin F, Polyakov AN, Sagaidak RN, et al. Attempt to produce element 120 in the $244 \mathrm{Pu}+58 \mathrm{Fe}$ reaction. Phys Rev C. (2009) 79:024603. doi: 10.1103/PhysRevC.79.024603

70. Hofmann S, et al. 2012 GSI Anuual Report GSI Report vol 2012-C1. (2011). p. 205.

71. Heusch B, Volant C, Freiesleben H, Chestnut RP, Hildenbrand KD, Puhlhofer F, et al. The reaction mechanism in the system $132 \mathrm{Xe}+56 \mathrm{Fe}$ at 5.73 $\mathrm{MeV} / \mathrm{u}$ : evidence for a new type of strongly damped collisions. $Z$ Phys A. (1978) 288:391. doi: 10.1007/BF01417723

72. Toke J, Bock R, Dai GX, Gobbi A, Gralla S, Hildenbrand KD, et al. Quasifission - The mass-drift mode in heavy-ion reactions. Nucl Phys A. (1985) 440:327. doi: 10.1016/0375-9474(85)90344-6

73. du Rietz R, Williams E, Hinde DJ, Dasgupta M, Evers M, Lin CJ, et al. Mapping quasifission characteristics and timescales in heavy element formation reactions. Phys Rev C. (2013) 88:054618. doi: 10.1103/PhysRevC.88.054618

74. Itkis MG, Bogachev AA, Itkis IM, Kliman J, Knyazheva GN, Kondratiev NA, et al. The processes of fusion-fission and quasi-fission of superheavy nuclei. Nucl Phys A. (2007) 787:150c. doi: 10.1016/j.nuclphysa.2006.12.026
75. Itkis IM, Kozulin EM, Itkis MG, Knyazheva GN, Bogachev AA, Chernysheva EV, et al. Fission and quasifission modes in heavy-ion-induced reactions leading to the formation of Hs. Phys Rev C. (2011) 83:064613. doi: 10.1103/PhysRevC.83.064613

76. Kozulin EM, Knyazheva GN, Novikov KV, Itkis IM, Itkis MG, Dmitriev $\mathrm{SN}$, et al. Fission and quasifission of composite systems with $\mathrm{Z}=108$ 120: transition from heavy-ion reactions involving $\mathrm{S}$ and $\mathrm{Ca}$ to $\mathrm{Ti}$ and Ni ions. Phys Rev C. (2014) 94:054613. doi: 10.1103/PhysRevC.94. 054613

77. Banerjee K, Hinde DJ, Dasgupta M, Simpson EC, Jeung DY, Simenel C, et al. Mechanisms suppressing superheavy element yields in cold fusion reactions. Phys Rev Lett. (2019) 122:232503. doi: 10.1103/PhysRevLett.122.232503

78. Dasgupta M, Hinde DJ, Rowley N, Stefanini AM. Measuring barriers to fusion. Annu Rev Nucl Part Sci. (1998) 48:401. doi: 10.1146/annurev.nucl.48.1.401

79. Hagino K, Takigawa N. Subbarrier fusion reactions and manyparticle quantum tunneling. Prog Theor Phys. (2012) 128:1061. doi: 10.1143/PTP.128.1061

80. Back BB, Esbensen H, Jiang CL, Rehm KE. Recent developments in heavy-ion fusion reactions. Rev Mod Phys. (2014) 86:317. doi: 10.1103/RevModPhys.86.317

81. Montagnoli G, Stefanini AM. Recent experimental results in sub- and near-barrier heavy-ion fusion reactions. Eur Phys J A. (2017) 53:169. doi: 10.1140/epja/i2017-12350-2

82. Wang N, Wu XZ, Li ZX, Liu M, Scheid W. Applications of Skyrme energy-density functional to fusion reactions for synthesis of superheavy nuclei. Phys Rev C. (2006) 74:044604. doi: 10.1103/PhysRevC.74. 044604

83. Wang B, Wen K, Zhao WJ, Zhao EG, Zhou SG. Systematics of capture and fusion dynamics in heavy-ion collisions. At Data Nucl. Data Tables. (2017) 114:281. doi: 10.1016/j.adt.2016. 06.003

84. Loveland W. An experimentalist's view of the uncertainties in understanding heavy element synthesis. Eur Phys J A. (2015) 51:120. doi: 10.1140/epja/i2015-15120-2

85. Bao XJ, Guo SQ, Zhang HF, Li JQ. Influence of proton shell closure on the evaporation residue cross sections of superheavy nuclei. J Phys G Nucl Part Phys. (2017) 44:045105. doi: 10.1088/1361-6471/aa53e8

86. Diaz-Torres A, Hinde DJ, Dasgupta M, Milburn GJ, Tostevin JA. Dissipative quantum dynamics in low-energy collisions of complex nuclei. Phys Rev C. (2008) 78:064604. doi: 10.1103/PhysRevC.78.064604

87. Sargsyan VV, Kanokov Z, Adamian GG, Antonenko NV, Scheid W. Capture process in nuclear reactions with a quantum master equation. Phys Rev C. (2009) 80:034606. doi: 10.1103/PhysRevC.80.034606

88. Sargsyan VV, Adamian GG, Antonenko NV, Scheid W, Zhang HQ. Effects of nuclear deformation and neutron transfer in capture processes, and fusion hindrance at deep sub-barrier energies. Phys Rev C. (2011) 84:064614. doi: 10.1103/PhysRevC.84.064614

89. Esbensen H, Stefanini AM. Influence of multiphonon excitations and transfer on the fusion of Ca+Zr. Phys Rev C. (2014) 89:044616. doi: 10.1103/PhysRevC.89.044616

90. Jiang CL, Rehm KE, Back BB, Esbensen H, Janssens VF, Stefanini AM, et al. Influence of heavy-ion transfer on fusion reactions. Phys Rev C. (2014) 89:051603(R). doi: 10.1103/PhysRevC.89.051603

91. Jia HM, Lin CJ, Yang F, Xu XX, Zhang HQ, Liu ZH, et al. Fusion of 32S+94Zr: further exploration of the effect of the positive Qxn value neutron transfer channels. Phys Rev C. (2014) 89:064605. doi: 10.1103/PhysRevC.89.064605

92. Rachkov VA, Karpov AV, Denikin AS, Zagrebaev VI. Examining the enhancement of sub-barrier fusion cross sections by neutron transfer with positive Q values. Phys Rev C. (2014) 90:014614. doi: 10.1103/PhysRevC.90.014614

93. Misicu S, Greiner W. Influence of hexadecupole deformation and role of orientation in capture reactions with $48 \mathrm{Ca}$ projectiles leading to superheavy nuclei. Phys Rev C. (2004) 69:054601. doi: 10.1103/PhysRevC.69.054601

94. Misicu S, Esbensen $H$. Hindrance of heavy-ion fusion due to nuclear incompressibility. Phys Rev Lett. (2006) 96:112701. doi: 10.1103/PhysRevLett.96.112701 
95. Wong CY. Interaction Barrier in charged-particle nuclear reactions. Phys Rev Lett. (1973) 31:766. doi: 10.1103/PhysRevLett.31.766

96. Stokstad RG, Switkowski ZE, Dayras RA, Wieland RM. Measurements of fusion cross sections for heavy-ion systems at very low energies. Phys Rev Lett. (1976) 37:888. doi: 10.1103/PhysRevLett.37.888

97. Rowley N, Satchler GR, Stelson PH. On the "distribution of barriers" interpretation of heavy-ion fusion. Phys Lett B. (1991) 254:25. doi: 10.1016/0370-2693(91)90389-8

98. Sargsyan VV, Adamian GG, Antonenko NV, Scheid W. Peculiarities of the sub-barrier fusion with the quantum diffusion approach. Eur Phys J A. (2010) 45:125. doi: 10.1140/epja/i2010-10978-x

99. Sargsyan VV, Adamian GG, Antonenko NV, Scheid W, Zhang HQ. Subbarrier capture with quantum diffusion approach: actinide-based reactions. Eur Phys J A. (2011) 47:38. doi: 10.1140/epja/i2011-11038-y

100. Sargsyan VV, Adamian GG, Antonenko NV, Scheid W, Zhang HQ. Role of neutron transfer in capture processes at sub-barrier energies. Phys Rev C. (2012) 85:024616. doi: 10.1103/PhysRevC.85.024616

101. Sargsyan VV, Adamian GG, Antonenko NV, Scheid W, Zhang HQ. Influence of neutron transfer in reactions with weakly and strongly bound nuclei on the sub-barrier capture process. Phys Rev C. (2012) 86:014602. doi: 10.1103/PhysRevC.86.01 4602

102. Wakhle A, Simenel C, Hinde DJ, Dasgupta M, Evers M, Luong DH, et al. Interplay between quantum shells and orientation in quasifission. Phys Rev Lett. (2014) 113:182502. doi: 10.1103/PhysRevLett.113.182502

103. Umar AS, Oberacker VE, Maruhn JA, Reinhard PG. Entrance channel dynamics of hot and cold fusion reactions leading to superheavy elements. Phys Rev C. (2010) 81:064607. doi: 10.1103/PhysRevC.81.064607

104. Simenel C, Hinde DJ, du Rietz R, Dasgupta M, Evers M, Lin CJ. Influence of entrance-channel magicity and isospin on quasi-fission. Phys Lett B. (2012) 710:607. doi: 10.1016/j.physletb.2012.03.063

105. Simenel C. Nuclear quantum many-body dynamics: from collective vibrations to heavy-ion collisions. Eur Phys J A. (2012) 48:152. doi: 10.1140/epja/i2012-12152-0

106. Oberacker VE, Umar AS, Simenel C. Energy dependence of potential barriers and its effect on fusion cross sections. Phys Rev C. (2014) 89:034611. doi: 10.1103/PhysRevC.89.034611

107. Umar AS, Oberacker VE, Simenel C. Shape evolution and collective dynamics of quasifission in the time-dependent Hartree-Fock approach. Phys Rev C. (2015) 92:024621. doi: 10.1103/PhysRevC.92.024621

108. Sekizawa K, Yabana K. Time-dependent Hartree-Fock calculations for multinucleon transfer and quasifission processes in the $64 \mathrm{Ni}+238 \mathrm{U}$ reaction. Phys Rev C. (2016) 93:054616. doi: 10.1103/PhysRevC.93.054616

109. Umar AS, Oberacker VE, Simenel C. Fusion and quasifission dynamics in the reactions $48 \mathrm{Ca}+249 \mathrm{Bk}$ and $50 \mathrm{Ti}+249 \mathrm{Bk}$ using a timedependent Hartree-Fock approach. Phys Rev C. (2016) 94:024605. doi: 10.1103/PhysRevC.94.024605

110. Umar AS, Simenel C, Ye W. Transport properties of isospin asymmetric nuclear matter using the time-dependent Hartree-Fock method. Phys Rev C. (2017) 96:024625. doi: 10.1103/PhysRevC.96.024625

111. Sekizawa K. Enhanced nucleon transfer in tip collisions of $238 \mathrm{U}+124 \mathrm{Sn}$. Phys Rev C. (2017) 96:041601(R). doi: 10.1103/PhysRevC.96.041601

112. Guo L, Simenel C, Shi L, Yu C. The role of tensor force in heavy-ion fusion dynamics. Phys Lett B. (2018) 782:401. doi: 10.1016/j.physletb.2018.05.066

113. Guo L, Godbey K, Umar AS. Influence of the tensor force on the microscopic heavy-ion interaction potential. Phys Rev C. (2018) 98:064607. doi: 10.1103/PhysRevC.98.064607

114. Simenel C, Umar AS. Heavy-ion collisions and fission dynamics with the time-dependent HartreeCFock theory and its extensions. Prog Part Nucl Phys. (2018) 103:19. doi: 10.1016/j.ppnp.2018.07.002

115. Godbey K, Umar AS, Simenel C. Deformed shell effects in Ca48+Bk249 quasifission fragments. Phys Rev C. (2019) 100:024610. doi: 10.1103/PhysRevC.100.024610

116. Bao XJ, Gao Y, Li JQ, Zhang HF. Theoretical study of the synthesis of superheavy nuclei using radioactive beams. Phys Rev C. (2015) 91:064612. doi: 10.1103/PhysRevC.91.064612

117. Bao XJ, Gao Y, Li JQ, Zhang HF. Influence of nuclear basic data on the calculation of production cross sections of superheavy nuclei. Phys Rev C. (2015) 92:014601. doi: 10.1103/PhysRevC.92.01 4601

118. Swiatecki WJ, Siwek-Wilczynska K, Wilczynski J. Fusion by diffusion. II. Synthesis of transfermium elements in cold fusion reactions. Phys Rev C. (2005) 71:014602. doi: 10.1103/PhysRevC.71.014602

119. Hagino K. Hot fusion reactions with deformed nuclei for synthesis of superheavy nuclei: an extension of the fusion-by-diffusion model. Phys Rev C. (2018) 98:014607. doi: 10.1103/PhysRevC.98.014607

120. Bao XJ, Guo SQ, Zhang HF, Li JQ. Dynamics of complete and incomplete fusion in heavy ion collisions. Phys Rev C. (2018) 97:024617. doi: 10.1103/PhysRevC.97.024617

121. Guo SQ, Gao Y, Li JQ, Zhang HF. Dynamical deformation in heavy ion reactions and the characteristics of quasifission products. Phys Rev C. (2017) 96:044622. doi: 10.1103/PhysRevC.96. 044622

122. Adamian GG, Antonenko NV, Scheid W. Characteristics of quasifission products within the dinuclear system model. Phys Rev C. (2003) 68:034601. doi: 10.1103/PhysRevC.68.034601

123. Volkov VV. The mechanism of compound nucleus formation in complete fusion of two massive nuclei. Phys Atom Nuclei. (2003) 66:1065. doi: 10.1134/1.1586419

124. Zagrebaev VI, Greiner W. Cross sections for the production of superheavy nuclei. Nucl Phys A. (2015) 944:257-307. doi: 10.1016/j.nuclphysa.2015.02.010

125. Diaz-Torres A. Modeling of compound nucleus formation in the fusion of heavy nuclei. Phys Rev C. (2004) 69:021603(R). doi: 10.1103/PhysRevC.69.021603

126. Diaz-Torres A. Competition between fusion and quasifission in a heavy fusing system: diffusion of nuclear shapes through a dynamical collective potential energy landscape. Phys Rev C. (2006) 74:064601. doi: 10.1103/PhysRevC.74.064601

127. Aritomo Y. Analysis of dynamical processes using the mass distribution of fission fragments in heavy-ion reactions. Phys Rev C. (2009) 80:064604. doi: 10.1103/PhysRevC.80.064604

128. Bao XJ. Possibility to produce $293,295,296 \mathrm{Og}$ in the reactions 48Ca+249,250,251Cf. Phys Rev C. (2019) 100:011601(R). doi: 10.1103/PhysRevC.100.011601

129. Schmidtt KH, Morawekt W. The conditions for the synthesis of heavy nuclei. Rep Prog Phys. (1991) 54:949-1003. doi: 10.1088/0034-4885/54/7/002

130. Ivanyuk FA, Ishizuka $\mathrm{C}$, Usang $\mathrm{MD}$, Chiba $\mathrm{S}$. Temperature dependence of shell corrections. Phys Rev C. (2018) 97:054331. doi: 10.1103/PhysRevC.97.054331

131. Zhu L, Wen PW, Lin CJ, Bao XJ, Su J, Li C, et al. Shell effects in a multinucleon transfer process. Phys Rev C. (2018) 97:044614. doi: 10.1103/PhysRevC.97.044614

132. Sauer G, Chandra H, Mosel U. Thermal properties of nuclei. Nucl Phys A. (1976) 264:221. doi: 10.1016/0375-9474(76)90429-2

133. Fröbrich P. On the dynamics of fission of hot nuclei. Nucl Phys A. (2007) 787:170c. doi: 10.1016/j.nuclphysa.2006.12.028

134. Brack M, Jens D, Jensen AS, Pauli HC, Strutinsky VM, Wong CY. Funny hills: the shell-correction approach to nuclear shell effects and its applications to the fission process. Rev Mod Phys. (1972) 44:320. doi: 10.1103/RevModPhys.44.320

135. Davies KTR, Sierk AJ, Nix JR. Effect of viscosity on the dynamics of fission. Phys Rev C. (1976) 13:2385. doi: 10.1103/PhysRevC.13.2385

136. Schunck N, Robledo LM. Microscopic theory of nuclear fission: a review. Rep Prog Phys. (2016) 79:116301. doi: 10.1088/0034-4885/79/11/ 116301

137. Schmidt KH, Jurado B. Review on the progress in nuclear fissionexperimental methods and theoretical descriptions. Rep Prog Phys. (2018) 81:106301. doi: 10.1088/1361-6633/aacfa7

138. Bao XJ. Possibilities for synthesis of new neutron-deficient isotopes of superheavy nuclei. Chin Phys C. (2019) 43:054105. doi: 10.1088/1674-1137/43/5/054105

139. Ignatyuk AV, Smirenkin GN, Tishin AS. Yad Fiz. (1975) 21:485. [Sov. J. Nucl. Phys. (1975) 29:255].

140. Vandenbosch R, Huizenga JR. Nuclear Fission. New York, NY: Academic (1973). 
141. Bao XJ, Guo SQ, Zhang HF, Li JQ. Influence of entrance channel on production cross sections of superheavy nuclei. Phys Rev C. (2017) 96:024610. doi: 10.1103/PhysRevC.96.024610

142. Liu ZH, Bao JD. Q-value effects on the production of superheavy nuclei. Phys Rev C. (2006) 74:057602. doi: 10.1103/PhysRevC.74.057602

143. Loveland W. Synthesis of transactinide nuclei using radioactive beams. Phys Rev C. (2007) 76:014612. doi: 10.1103/PhysRevC.76.014612

144. Loveland W. The synthesis of new neutron-rich heavy nuclei. Front Phys. (2019) 7:23. doi: 10.3389/fphy.2019. 00023
Conflict of Interest: The author declares that the research was conducted in the absence of any commercial or financial relationships that could be construed as a potential conflict of interest.

Copyright (C) 2020 Bao. This is an open-access article distributed under the terms of the Creative Commons Attribution License (CC BY). The use, distribution or reproduction in other forums is permitted, provided the original author(s) and the copyright owner(s) are credited and that the original publication in this journal is cited, in accordance with accepted academic practice. No use, distribution or reproduction is permitted which does not comply with these terms. 